\title{
Generating anagrams from multiple core strings employing user-defined vocabularies and orthographic parameters
}

\author{
TIMOTHY R. JORDAN and AXEL MONTEIRO \\ University of Nottingham, Nottingham, England
}

\begin{abstract}
Anagrams are used widely in psychological research. However, generating a range of strings with the same letter content is an inherently difficult and time-consuming task for humans, and current computerbased anagram generators do not provide the controls necessary for psychological research. In this article, we present a computational algorithm that overcomes these problems. Specifically, the algorithm processes automatically each word in a user-defined source vocabulary and outputs, for each word, all possible anagrams that exist as words (or as nonwords, if required) as defined by the same source vocabulary. Moreover, we show how the output of the algorithm can be filtered to produce anagrams within specific user-defined orthographic parameters. For example, the anagrams produced can be filtered to produce words that share, with each other or with other words in the source vocabulary, letters in only certain positions. Finally, we provide free access to the complete Windows-based program and source code containing these facilities for anagram generation.
\end{abstract}

Anagrams play an important and pervasive role in psychological research. For example, anagrams provide a measure of problem-solving ability (i.e., where the task is to generate a word composed of the same letters as a presented string) that has been used to study a range of psychological issues, including insight (e.g., Smith \& Kounios, 1996), aging (e.g., Witte \& Freund, 1995), recognition memory (e.g., Weldon, 1991), semantic memory (e.g., White, 1988), and the topography of evoked brain activity (e.g., Skrandies, Reik, \& Kunze, 1999). Anagrams have also been used extensively to study processes involved in word recognition, where a great deal of research involves comparing performances between stimuli from different linguistic categories, including frequency, imageability, concreteness, orthographic structure, and lexicality (words vs. nonwords). ${ }^{1}$ For example, when performance with words and nonwords was compared, several studies (e.g., Gibson, Pick, Osser, \& Hammond, 1962; Jordan, Patching, \& Milner, 2000; Mason, 1975; Massaro \& Klitzke, 1979; Massaro, Venezky, \& Taylor, 1979; Reicher, 1969) have used stimuli matched for their individual letter content (e.g., show vs. ohws). The attraction of this matching is that differences in basic letter content can be removed as a confounding variable between categories and so allow influ-

This work was supported by BBSRC Grant 42/S12111 to T.R.J. The order of authorship is alphabetical, and both authors contributed equally to this work. Correspondence concerning this article should be addressed to either T. R. Jordan or A. Monteiro, School of Psychology, University of Nottingham, University Park, Nottingham NG7 2RD, England (e-mail: trj@psychology.nottingham.ac.uk or lpxaxm@psychology. nottingham.ac.uk). ences of linguistic category (e.g., frequency, imageability, concreteness, orthographic structure, lexicality) on performance to be revealed more clearly.

The appropriate selection of core strings (e.g., slate) and the generation of anagrams (in this case, including the words least, stale, steal, tales, teals, and tesla) for use in any psychological research requires certain controls over the procedure of core string selection and anagram generation that will avoid confounds that may contaminate the data produced by an experiment. First, control over the source vocabulary used to validate (as legal words) core strings and their letter-string permutations ensures that only linguistically appropriate core strings and anagrams are used. Most obviously, defining the language within which core strings exist and from which anagrams are generated ensures that all permutations are relevant for a particular participant population. However, this is important not only for determining core strings and anagrams for languages that are highly individual (e.g., English vs. French), but also for core strings and anagrams specific to variations within a language; for example, despite their overall similarity, American, Australian, British, and Canadian English contain words that vary in their presence, spelling, and frequency of usage across these three vocabularies. In addition, control over the source vocabulary allows anagrams to be produced from either an exhaustive search of the entire vocabulary of a language or a subset of the vocabulary (e.g., one including only words above a certain frequency of usage).

Second, the selection of core strings and anagrams for an experiment is facilitated and its validity enhanced when all the anagrams of all the words in the chosen vocabulary are available at the start of the selection process. 
Without this availability, the appropriateness of core strings and their anagrams for inclusion in an experiment is difficult to determine, and the entire selection process is susceptible to experimenter bias. For example, core words that are subjectively more likely to be generated (e.g., of higher frequencies of occurrence) are more likely to be selected. Indeed, knowing the number of real-word anagrams that can be produced from a particular core string and how this compares with the number produced by other, potential core strings is crucial for assessing the suitability of a core string for a particular task. For example, when nonword anagrams are selected for use in problem-solving experiments or as controls in word recognition experiments, nonwords with just one real-word anagram would provide qualitatively different stimuli, as compared with those for which two or more real-word anagrams exist.

Third, control over the nature of the anagrams generated allows a more refined and focused use of anagrams in psychological research. In particular, when anagrams of core strings are generated, user-defined constraints placed on the operation of the generating algorithm allow only the types of anagram relevant to the aims of an experiment to be specified and produced and so avoid the production of all (including unwanted) combinations that satisfy the general principle of an anagram: For example, anagrams above a certain frequency of written occurrence, anagrams for which letters in only certain positions in the core word are transposed, or anagrams that share letters or groups of letters in only certain positions with other words in the source vocabulary.

However, generating a range of different strings with the same letter content is an inherently difficult and time-consuming task (at least for humans), particularly when each permutation must be verified as a word or a nonword by checking for its existence in the appropriate vocabulary. Indeed, whereas the generation and verification of all possible permutations is feasible for a single core string of three or four letters, the generation and verification of all possible permutations for multiple core strings, especially those of five letters or more, demands computational involvement to achieve acceptable levels of accuracy, efficiency, and validity.

Several computer-based anagram generators are available, and each takes in a single word, phrase, or sentence and transposes the letters to produce a new legal English word, phrase, or sentence. Many of these generators are implemented over the Internet and can be freely accessed using standard Web browsers, such as Internet Explorer and Netscape Navigator (see Appendix A). Several others are available as freeware or shareware programs and can be downloaded from the Internet and used either for free or on payment of a nominal fee. Commercial anagramgenerating programs are also available and generally offer more sophisticated anagram-generating algorithms, but at a greater cost (see Appendix B).

However, irrespective of their source, none of these anagram generators are well suited to the production of experimental stimuli for psychological research, because they fail on at least two of the three controls outlined ear- lier (see Table 1)—more specifically, with respect to the following.

1. The vast majority of anagram generators currently available provide no facility for determining the system's source vocabulary (used to verify the core letter-string and its permutations as legal words), and most provide no indication of the source used. This is unsuitable for the production of experimental stimuli, for which knowledge and control of the nature of the source vocabulary is required to produce a stimulus set of known characteristics and maximal ecological validity. In particular, an inappropriate source vocabulary may not provide anagrams representative of the linguistic environment of participants. Indeed, problems arise not only when the source vocabulary has an unknown content, but also when the content is known but is less than ideal-for example, when it contains too few words or contains spellings that are inappropriate for a particular participant population (e.g., American English for British participants).

2. All anagram generators currently available allow the production of anagrams from core letter strings only by taking as input one core string at a time. This input string must then be processed before the next input string can be entered by the researcher. This piecemeal approach is unsuited to the production of experimental stimuli, where sufficient numbers of appropriately matched stimuli are likely to be achieved only after permutations from several thousand input strings have been calculated.

3. No anagram generator currently available allows any user-defined, research-relevant constraints to be placed on the operation of the generating algorithm. Thus, when anagrams of core strings are derived, all combinations that satisfy the general principle of an anagram are generated without allowing constraints on such things as the frequency of occurrence or the orthographic structure of the strings produced.

In this article, we present a computational algorithm for producing anagrams from any suitable user-defined source vocabulary. Specifically, the algorithm takes in each word in a chosen vocabulary and outputs, for each word, all possible anagrams that exist in the same vocabulary (and, if required, all that do not-i.e., nonwords). Moreover, the output of the algorithm can be filtered to produce anagrams within specific user-defined orthographic parameters. For example, the anagrams produced can be filtered to produce words that share, with each other or with other words in the source vocabulary, letters in only certain positions.

We present a design for a computer program that can take in large numbers of words (e.g., from any suitable source vocabulary) in a single submission (i.e., without individual word input) and can produce anagrams for each word within user-defined constraints. By providing this information, our intention is to allow the algorithm to be implemented within the bespoke programs of researchers to produce strings specific to the demands of individual experiments. To date, we have implemented the algorithm in $\mathrm{C} / \mathrm{C}++$ and verified its use with a number of substantial vocabularies readily and freely available in electronic format, including the MRC Psycholinguis- 
Table 1

Limitations of Current Computer-Based Anagram Generators

\begin{tabular}{|c|c|c|c|}
\hline Generator & $\begin{array}{c}\text { Dictionary } \\
\text { Control }\end{array}$ & $\begin{array}{l}\text { Multiple Core } \\
\text { Input Strings }\end{array}$ & $\begin{array}{c}\text { User-Def ined } \\
\text { Output Constraints }\end{array}$ \\
\hline \multicolumn{4}{|c|}{ On-Line Anagram Generators } \\
\hline The WWW Anagram Generator & no & no & no \\
\hline Brendan's On-Line Anagram Generator & no & no & no \\
\hline Internet Anagram Server & no & no & no \\
\hline Andy's Anagram Solver & no & no & no \\
\hline The Anagram Engine & no & no & no \\
\hline Arrak Anagrams & no & no & no \\
\hline Inge's Anagram Generator & no & no & no \\
\hline Anagram Dictionary & no & no & no \\
\hline Martin's Anagram Generator & no & no & no \\
\hline Hiten Sonpal’s Anagrams & no & no & no \\
\hline Jumbles Unjumbled & no & no & no \\
\hline \multicolumn{4}{|c|}{ Freeware and Shareware Anagram Generators } \\
\hline Winagram 1.0 & yes & no & no \\
\hline Logos (http://homepage.ntlworld.com/jeremy.riley/Logos) & no & no & no \\
\hline Anagram Generator 1.19 & yes & no & no \\
\hline The Electronic Alveary 1.6 & no & no & no \\
\hline Anagrams 2.0 & yes & no & no \\
\hline ABC Genius (http://www.bebesoft.com) & yes & no & no \\
\hline TeaBag 2.0 (http://www.tiac.net/users/hlynka/anagrams) & yes & no & no \\
\hline PuzzLex 5.11 (http://www.puzzlex.co.uk) & no & no & no \\
\hline \multicolumn{4}{|c|}{ Commercial Anagram Generators } \\
\hline Anagram Genius 8.0 & yes & no & no \\
\hline
\end{tabular}

tic Database (http://www.psy.uwa.edu.au/MRCDataBase/mrc2.html), the Carnegie Mellon Pronouncing Dictionary (http://www.speech.cs.cmu.edu/cgi-bin/cmudict), the Moby lexicon (http://www.dcs.shef.ac.uk/research/ ilash/Moby), and the Natural Language wordlists at DEC (ftp://gatekeeper.dec.com/pub/misc/stolfi-wordlists/ english.tar.Z). In addition, the algorithm can also be readily applied to the file/usr/dict/words, supplied as part of most Unix systems.

\section{The Anagram Algorithm}

The general design of a program to determine anagrams for a specific vocabulary is as follows:

Start: Read the vocabulary into a data structure in memory.

Iterate through the core strings in the vocabulary, for each core string:

Output the core string.

Calculate all permutations for letters of the current word.

Check each permutation against the input vocabulary. Discard any permutations that do not appear in the input vocabulary.

Output the permutations for this word.

Exit: Finished processing all the core strings in the vocabulary. Apply any further filtering as necessary (e.g., discard permutations that do not contain particular letters in certain serial positions).

The above design outlines the major components of a program for determining all anagrams for a given source vocabulary. The program begins by reading a target vocabulary into an appropriate data structure held in memory. Once this has been achieved, all core strings in the vocabulary are processed one by one. For each core string, all possible permutations of the letters of the core string are determined, and then each of these permutations is validated as a legal word by checking against the source vocabulary. An implementation of the above anagram algorithm is relatively straightforward to produce. However, the overall efficiency (and utility) of the complete implemented program is highly dependant on the specific implementation of the algorithm component that determines the permutations for each core string.

Determining all of the permutations for each core string can be achieved in several ways. However, since vocabularies are likely to contain words of different lengths, it is desirable that the implementation should be capable of producing permutations for all core strings in the vocabulary, regardless of differences in their lengths. If this is not the case, the vocabulary must be divided into subvocabularies according to core string length. Each subvocabulary must then be processed individually, and the implementation of the anagram program must be adjusted for each subvocabulary (i.e., for each core string length required).

Producing the permutations so that the same procedure is implemented regardless of word length is the most algorithmically complex component of an anagramgenerating program. We will therefore present this lower level permutations algorithm in more detail. (An implementation of the permutations algorithm as a $\mathrm{C} / \mathrm{C}++$ function is provided in Appendix C.) 


\section{The Permutations Algorithm}

The total number of permutations for a core string composed of $N$ letters is given by $N$ ! (i.e., $N$-factorial). Therefore, if a core string is four letters long, there is a total of 24 permutations of the letters of that core string (e.g., 4 ! = $4 \times 3 \times 2 \times 1=24)$. There are several ways to compute all 24 of these permutations. However, as was mentioned above, it is advantageous for a single procedure to be employed that is capable of determining all of the permutations for any core string, regardless of its length.

Therefore, the algorithm described here determines permutations in a recursive manner. It begins by finding all permutations based on the first letter in the first letter position (with the remaining letters and letter positions unchanged). The only possible permutation for this is the original sequence of letters from the input core string. This permutation is then used with the second letter and the second letter position to determine all permutations of the core string in which only the first two letters have been transposed around the first two letter positions (i.e., the rest of the letters remain in their original positions). These permutations are then used with the third letter and the third letter position to find all permutations of the core string involving transposition of only the first three letters around the first three letter positions. This set of permutations is then used with the fourth letter and the fourth letter position to calculate all permutations based on the first four letters and the first four letter positions of the core string. This process is repeated (i.e., the $N$ th letter of the word and the $N$ th letter position are used with the previously determined set of permutations), until all the letters of the core string have been processed and the complete set of permutations for the core string has been produced.

The procedure performed by the permutations algorithm is essentially the following:

Start: $\quad$ Set $K$ to 0 .

Loop1: $\quad$ Add 1 to $K$.

Set $n$ to 0 .

Loop 2: $\quad$ Add 1 to $n$.

Take the letter in position $K$ and make a new permutation by swapping it with the letter in position $K-n$ for every possible permutation based on $K-1$ letters.

If $n$ is less than $K$ then go back to Loop2.

If $K=$ the number of letters in the string then go to Exit.

If $n=\mathrm{K}$ then go back to Loop 1 .

Exit: Finished calculating all the permutations for this string.

For a four-letter core string this works as follows: Starting with $K=1$ (i.e., the first letter of the core string), there are no swaps to make since there can be no permutations based on $K-1$ letters (when $K=1, K-1$ is letter position zero; hence, no permutations). The only possible permutation involving the first letter with the first letter position is the initial core string itself. So, for a four-letter core string $\mathrm{ABCD}$, when $K=1$, the list of permutations is equal to

\section{$\mathrm{ABCD}$}

When $K=2$, the letter in Position 2 (i.e., B), is swapped with the letter in Position 1 in all permutations based on Letter Position 1. This gives the following set of permutations:

\section{$\mathrm{ABCD}$ \\ $\mathrm{BACD}$}

When $K=3$, the third letter, $\mathrm{C}$, is swapped with all letters in Position 2, and all letters in Position 1 in every permutation determined so far (i.e., both of those above), giving
$\mathrm{ABCD}$
BACD
CBAD
$\mathrm{ACBD}$
CABD
$\mathrm{BCAD}$

Finally (for four-letter words), $K=4$, letter D must be swapped in Letter Positions 1, 2, and 3 in the above, giving

$\begin{array}{llll}\text { ABCD } & \text { DBCA } & \text { DBAC } & \text { DABC } \\ \text { BACD } & \text { ADCB } & \text { CDAB } & \text { CDBA } \\ \text { CBAD } & \text { ABDC } & \text { CBDA } & \text { CADB } \\ \text { ACBD } & \text { DACB } & \text { DCBA } & \text { DCAB } \\ \text { CABD } & \text { BDCA } & \text { ADBC } & \text { BDAC } \\ \text { BCAD } & \text { BADC } & \text { ACDB } & \text { BCDB }\end{array}$

Hence, the complete set of permutations for the core string ABCD is derived. Further components of the anagram algorithm can now check these permutations against the source vocabulary (i.e., discarding permutations that do not appear in the source vocabulary if valid words are required, or discarding permutations that $d o$ appear in the source vocabulary if nonwords are required) and can perform any further filtering according to the specific requirements of experiments. For example, a filter can be applied to the output of the above algorithm that compares the letter positions of each permutation with those of the initial core string. Permutations can then be accepted or rejected on the basis of transposed letter content. In this way, large quantities of letter-string stimuli can be produced easily from any appropriate source vocabulary to satisfy the requirements of virtually any psychological experiment in which anagram stimuli are required.

An implementation of the permutations algorithm above in $\mathrm{C} / \mathrm{C}++$ is provided in Appendix $\mathrm{C}$. We have developed a complete anagram-generating program based on the previously described anagram algorithm that also incorporates the permutations algorithm above. The complete Windows-based program, the source code, and as- 
sistance with development and implementation of filters are available free of charge from the authors. Please e-mail lpxaxm@psychology.nottingham.ac.ukfor further details.

\section{REFERENCES}

Gibson, E. J., Pick, A., Osser, H., \& Hammond, M. (1962). The role of grapheme-phoneme correspondence in the perception of words. American Journal of Psychology, 75, 554-570.

Jordan, T. R., Patching, G. R., \& Milner, A. D. (2000). Lateralized word recognition: Assessing the role of hemispheric specialization and perceptual asymmetry. Journal of Experimental Psychology: Human Perception \& Performance, 26, 1192-1208.

Mason, M. (1975). Reading ability and letter search time: Effects of orthographic structure defined by single-letter positional frequency. Journal of Experimental Psychology: General, 104, 146-166.

Massaro, D. W., \& KlitzKe, D. (1979). The role of lateral masking and orthographic structure in letter and word recognition. Acta Psychologica, 43, 413-426.

Massaro, D. W., Venezky, R. L., \& Taylor, G. A. (1979). Orthographic regularity, positional frequency, and visual processing of letter strings. Journal of Experimental Psychology: General, 108, 107-124.

ReICHER, G. M. (1969). Perceptual recognition as a function of mean- ingfulness of stimulus material. Journal of Experimental Psychology, 81, 275-280.

Skrandies, W., ReIK, P., \& Kunze, C. (1999). Topography of evoked brain activity during mental arithmetic and language tasks: Sex differences. Neuropsychologia, 37, 421-430.

Smith, R. W., \& Kounios, J. (1996). Sudden insight: All-or-none processing revealed by speed-accuracy decomposition. Journal of Experimental Psychology: Learning, Memory, \& Cognition, 26, 1443-1462. WeLDON, M. S. (1991). Mechanisms underlying priming on perceptual tasks. Journal of Experimental Psychology: Learning, Memory, \& Cognition, 17, 526-541.

White, H. (1988). Semantic priming of anagram solutions. American Journal of Psychology, 101, 383-399.

WitTe, K. L., \& Freund, J. S. (1995). Anagram solution as related to adult age, anagram difficulty, and experience in solving crossword puzzles. Aging \& Cognition, 2, 146-155.

\section{NOTE}

1. Grammatically, the term anagram refers to words derived from other words, but not to nonwords derived from any type of string. However, for simplicity, we do not make this distinction when using the term anagram in this article.

\section{APPENDIX A}

\section{Web Browsers}

Internet Explorer 6.0 is available free of charge from Microsoft Corporation at http://www.microsoft.com. Netscape Navigator 6.0 is available free of charge from Netscape Communications at http://www.netscape.com.

\section{On-Line Anagram Generators}

The WWW Anagram Generator, by Eli Burke, is available for use free of charge at http://www.failte.demon. co.uk/anagrams.htm.

Brendan's On-Line Anagram Generator, by Brendan Connell, is available for use free of charge at http://www.mbhs.edu/ bconnell/anagrams.html.

Internet Anagram Server, by Anu Garg, is available for use free of charge at http://www.wordsmith.org/anagram.

Andy's Anagram Solver, by Andrew M. Gay, is available for use free of charge at http:// www.ssynth.co.uk/ $\sim$ gay/anagram.html.

The Anagram Engine, by EasyPeasy Ltd., is available for use free of charge at http://www.easypeasy.com/anagrams/index.html.

Arrak Anagrams, by Arrak Software, is available for use free of charge at http://ag.arrak.fi/index_en.html.

Inge's Anagram Generator, by Inge Kristian Eliassen, is available for use free of charge at http://www.mi.uib.no/ ingeke/anagram.

Anagram dictionary, by Luke Metcalfe, is available for use free of charge at http://www.orchy.com/dictionary/ anagrams.htm.

Martin's Anagram Generator, by Martin Mamo, is available for use free of charge at http://freespace.virgin.net/ martin.mamo/anagram.html.

Hiten Sonpal's Anagrams, by Hiten Sopal, is available for use free of charge at http://spruce.evansville.edu/ $\sim$ hs4/anagrams.

Jumbles Unjumbled, by Brian R. Owen, is available for use free of charge at http://www.eecg. toronto.edu/ bryn/HTML/Jumbles.html. 


\section{APPENDIX B}

\section{Freeware and Shareware Anagram Programs}

Winagram 1.0, by Jenies Technologies Incorporated, is available free and can be downloaded from http://eyenettools.com/Anagram.htm.

Logos, by Jeremy Riley, is available free and can be downloaded from http://homepage.ntlworld.com/jeremy. riley/Logos.

Anagram Generator 1.19, by Jason Rampe and Jack Rampe, is available free and can be downloaded from http://fractalchaos.freeyellow.com/anagram.htm.

The Electronic Alveary 1.6, by Ross Beresford, is available for $£ 25$ (single-user license) from Bryson Limited, 10 Wagtail Close, Twyford, Reading RG10 9ED, UK. An evaluation version can be downloaded from http://www.bryson.demon.co.uk.

Anagrams 2.0, by Andrew Trevorrow, is available for \$15 (single-user license), and can be downloaded from http://www.trevorrow.com/anagrams/index.html.

$\mathrm{ABC}$ genius, by Be-Best Software, is available for $\$ 20$. A free evaluation version can be downloaded from http://www.bebesoft.com.

TeaBag 2.0, by Adrian Hlynka, is available free and can be downloaded from http://www.tiac.net/ users/hlynka/anagrams.

PuzzLex 5.11, by N. Bentley, is available for $£ 15$. A free evaluation version can be downloaded from http://www.puzzlex.co.uk.

\section{Commercial Anagram Programs}

Anagram Genius 8.0, by Genius 2000 Software, is available for $£ 24.99$ from Genius 2000 Software at http://www.genius2000.com/ag.html.

\section{APPENDIX C}

The implementation of the permutations algorithm as a $\mathrm{C} / \mathrm{C}++$ function is presented below. The function takes as arguments: the number of letters in the word, a pointer to an area of memory (referenced as a twodimensional (2-D) array of char) big enough to hold all of the permutations and an int that specifies how many permutations there will be in total (i.e., how many rows in the 2-D array, calculated by $N !$, where $N$ is the number of letters in the input word).

The word to be processed is supplied in row zero of the permutations array. New anagrams are calculated by copying appropriate (previously calculated) anagrams to the next empty row in the array and then swapping the appropriate letters.

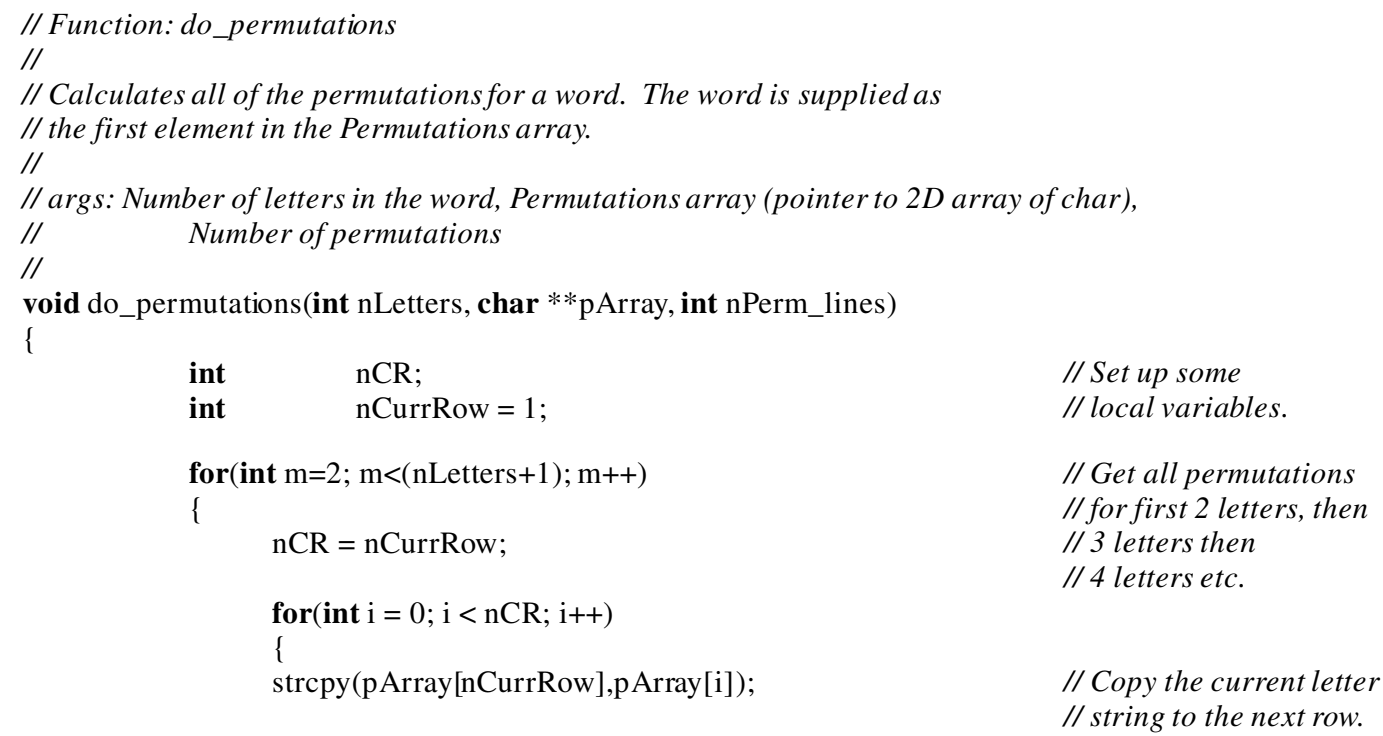


APPENDIX C

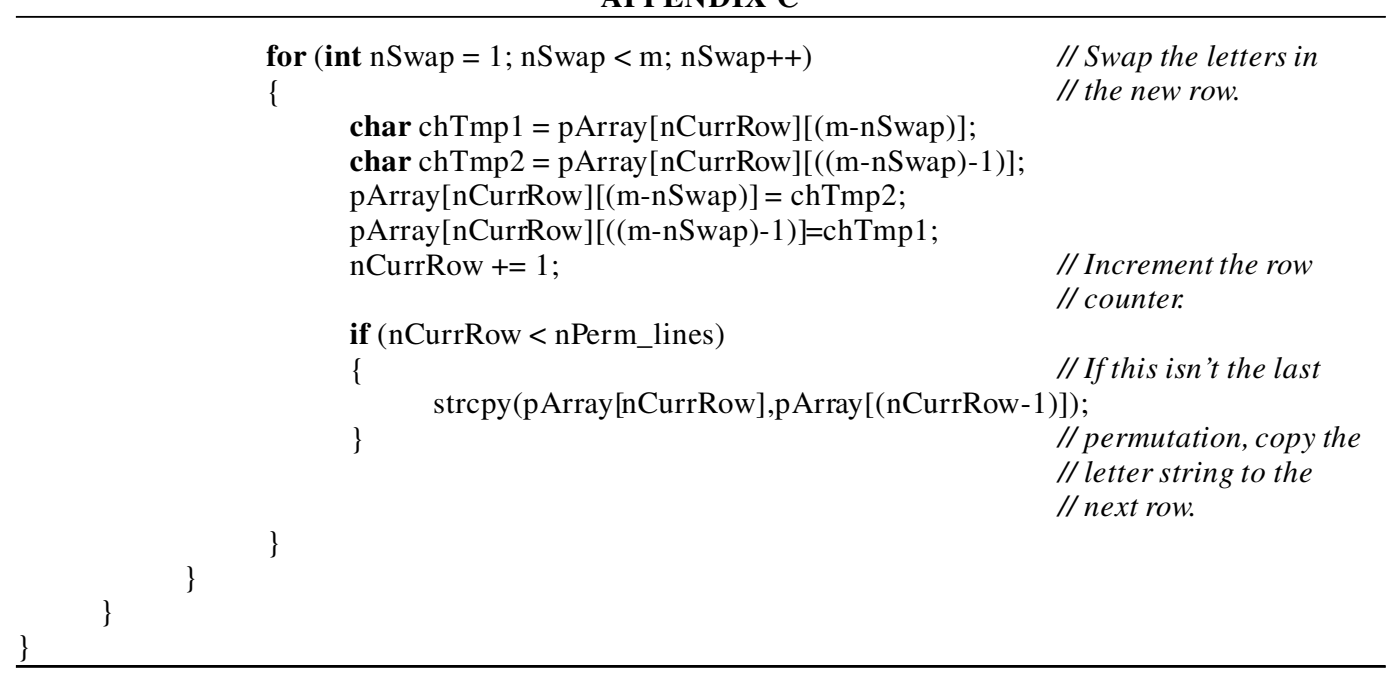

(Manuscript received July 25, 2001; revision accepted for publication July 8, 2002.) 